\title{
Effects of Aerobic Exercise on Patients with Mental Illness in a Veterans Inpatient Psychiatric Unit: A Review of the Literature
}

\author{
John Allen McCash ${ }^{1} \&$ Sam Abraham ${ }^{2}$ \\ ${ }^{1}$ Battle Creek Veterans Affairs Medical Center, Battle Creek, Michigan, USA \\ ${ }^{2}$ Bethel College School of Nursing, Mishawaka. Indiana, USA \\ Correspondence: Sam Abraham, Assistant Professor of Nursing, 1001 Bethel Circle, Bethel College School of \\ Nursing, Mishawaka, Indiana, 46545, USA.
}

Received: November 15, 2017

Accepted: December 2, 2017 Online Published: December 6, 2017

doi:10.20849/ijsn.v3i1.235

URL: https://doi.org/10.20849/ijsn.v3i1.235

\begin{abstract}
Mental illness continues to be a leading cause of stressor especially for veterans in the United States. These problems are affecting the role functioning and relationships. The purpose of this review was to explore extensive literature on the benefits of aerobic exercise that lead to non-pharmacological interventions to help patients who suffer from mental illness in a veterans hospital inpatient mental health unit. Patients admitted to inpatient mental health units have a diagnosis such as schizophrenia, bipolar disorder, major depression, or schizoaffective disorders. They should also meet the diagnostic criteria of suicidal or homicidal ideation. Sometimes it may be a veteran who is unable to care for self or needs medical supervision. Upon conclusion of the literature review, evidence has shown to provide significant data supporting the benefit of aerobic exercises.
\end{abstract}

Keywords: aerobic exercises, mental illness, veterans, inpatient mental health unit

\section{Introduction}

A review of the literature was conducted to investigate the benefits of introducing aerobic exercise to veterans in an inpatient mental health unit and the possibility of reducing symptoms of mental illness. The World Health Organization ([WHO], 2003) reports indicate that mental health is a growing issue worldwide, with more than 450 million people suffering from a mental illness. Schizophrenia and major depressive disorders are in the top 10 mental illness diagnoses (Oertel-Knochel et al., 2014) and is known to effect role functioning and relationships negatively for both the individual, loved ones, and society (Mojtabai, 2011). Exercise may help the veteran reduce symptoms of mental illness (Carter, Callaghan, Khalil, \& Morres, 2012).

Aerobic exercise can be used to help reduce psychopathological or abnormal behavioral symptoms while improving cognitive ability in people with mental illness (Oertel-Knochel et al., 2014). Physical exercise has been shown to increase neurogenesis and develop neuroplasticity and therefore increasing cognitive functioning (Oertel-Knochel et al., 2014). Furthermore, aerobic exercise has been shown to increase the prevalence of important growth factors including glucocorticoids, brain-derived neurotrophic factor (BDNF), insulin-like growth factor-1 (IGF-1) and vascular endothelial growth factor ([VEGF], Oertel-Knochel et al., 2014). Growth factors such as VEGF are essential in the growth of new capillaries to increase neuroplasticity that positively affects cognitive ability and mood that will, in turn decrease the symptoms of mental illness. Many people suffer from mental illness and veterans are at high risk for mental illness (Pols \& Oak, 2007).

Veterans are prone to mental health issues with exposure to combat, conflict, death, carnage, chronic pain, and separation from loved ones (Vazan, Golub, \& Bennett, 2013). Participating in multiple acts that are threatening to one's life can potentially lead to mental health problems such as in the veterans (Pols \& Oak, 2007). Aerobic exercise could help veterans better cope with their mental health disorders, be more productive in their lives, their relationships with others, and in the society.

Veterans on an inpatient mental health unit may benefit from regular aerobic exercise as an adjunct intervention to their therapy. On an inpatient unit, most of the veteran's day is filled with therapy, eating, and sleeping leading to a sedentary lifestyle. Physical exercise could be used as an intervention to distract the veteran and teach effective coping strategy for dealing with mental illness. Therefore, the purpose of this literature review was to explore extensive literature on the benefits of aerobic exercise that lead to non-pharmacological interventions, to help veterans who suffer from mental illness in an inpatient mental health setting. 


\section{Method}

The searches involved using the databases of Elsevier Science Direct Freedom Collection, ProQuest Central, Gale Academic OneFile, Cengage Learning, ScienceDirect, and Springer Standard Collection to find the primary peer-reviewed research articles to examine for this review of the literature ([ROL], see Table 1). Keywords used to search the literature included aerobic exercises; mental illness; veterans; and inpatient mental health unit. This search resulted in 325 peer-reviewed articles. An analysis was conducted of the titles to consider for abstract review based on the preliminary assessment, and 297 articles were excluded. The abstracts were reviewed for inclusion criteria. Qualifiers included severe mental illnesses. An additional exclusion of 18 articles was executed in an effort to balance the representation of mental illness diagnoses because of an abundance of research in schizophrenia. This left the search results with 10 valid articles for review.

Table 1. Search strategy and limiters

\begin{tabular}{|c|c|c|c|c|}
\hline Database Searched & $\begin{array}{l}\text { Date } \\
\text { Search }\end{array}$ & Search Strategy and Limiters & $\begin{array}{l}\text { Number of } \\
\text { Articles } \\
\text { Found }\end{array}$ & $\begin{array}{l}\text { Estimate of } \\
\text { Relevant } \\
\text { Articles }\end{array}$ \\
\hline Academic & $03 / 26 / 2017$ & $\begin{array}{l}\text { Key words: Relationships between } \\
\text { physical activity; Severe mental } \\
\text { illness; Inpatient; Quality of life } \\
\text { Limitations: Last } 5 \text { years; English; } \\
\text { Peer-reviewed journals }\end{array}$ & 16 & 2 \\
\hline $\begin{array}{l}\text { Springer } \\
\text { Collection }\end{array}$ & $02 / 19 / 2017$ & $\begin{array}{l}\text { Key words: Severe mental illness; } \\
\text { Client and clinician perspectives; } \\
\text { Walking group } \\
\text { Limitations: Last year; English; } \\
\text { articles }\end{array}$ & 28 & 1 \\
\hline $\begin{array}{l}\text { ProQuest Agricultural } \\
\& \text { Environmental } \\
\text { Science Database }\end{array}$ & 03/28/2017 & $\begin{array}{l}\text { Key words: Effects of aerobic } \\
\text { exercise; Mental illness; Intensity } \\
\text { exercise; Preferred } \\
\text { Limitations: Last } 5 \text { year; English; } \\
\text { articles; Depression }\end{array}$ & 36 & 3 \\
\hline Cengage Learning, Inc. & 03/28/2017 & $\begin{array}{l}\text { Key words: Effects of aerobic } \\
\text { exercise; Mental illness; Intensity } \\
\text { exercise; Preferred } \\
\text { Limitations: Last year; English; } \\
\text { Articles; Physical activity }\end{array}$ & 40 & 4 \\
\hline $\begin{array}{l}\text { Cumulative Index to } \\
\text { Nursing and Allied } \\
\text { Health } \\
\text { (CINAHL) }\end{array}$ & $01 / 27 / 2017$ & $\begin{array}{l}\text { Key words: Exercise and mental } \\
\text { health qualitative; } \\
\text { Limitations: } 2010-2016 \text {; Linked full } \\
\text { text; Academic journals }\end{array}$ & 20 & 4 \\
\hline ProQuest Central & $03 / 28 / 2017$ & $\begin{array}{l}\text { Key words: Mental health or illness; } \\
\text { Aerobic exercise; Circuit training; } \\
\text { Severe mental illness; Effects of } \\
\text { aerobic exercise; Intensity exercise; } \\
\text { Preferred } \\
\text { Limitations: Last } 5 \text { years; English; } \\
\text { Schizophrenia; Physical activity }\end{array}$ & 85 & 6 \\
\hline $\begin{array}{l}\text { Elsevier Science Direst } \\
\text { Freedom Collection }\end{array}$ & 03/28/2017 & $\begin{array}{l}\text { Key words: Mental health or illness; } \\
\text { Aerobic exercise; Circuit training; } \\
\text { Severe mental illness; Effects of } \\
\text { aerobic exercise; Intensity exercise; } \\
\text { Preferred } \\
\text { Limitations: Last } 5 \text { years; English; } \\
\text { Schizophrenia; Physical activity; } \\
\text { depression }\end{array}$ & 85 & 6 \\
\hline $\begin{array}{l}\text { ScienceDirect (Elsevier } \\
\text { B.V.) }\end{array}$ & 03/28/2017 & $\begin{array}{l}\text { Key words; Effects of aerobic } \\
\text { exercise; PTSD; Mental health; } \\
\text { Promotion } \\
\text { Limitations: Last year; English; }\end{array}$ & 15 & 2 \\
\hline
\end{tabular}




\section{Findings}

\subsection{Methods and Designs of Studies}

The findings from the review focused on 10 peer-reviewed articles. Frameworks of the reviewed articles were not specifically listed in the literature. However, the articles were found to be a collection of different methods and designs. Designs ranging from qualitative studies (Firth et al., 2016; McArdle, McGale, \& Gaffney, 2012) to quantitative (Bonsaksen \& Lerdal, 2012; Oretel-Knochel et al., 2014; Pfaff et al., 2014; Powers et al., 2015; Strassnig et al., 2015; Subramaniapillai et al., 2016; Toups et al., 2017) and a mix-method design (Browne, Mihas, \& Penn, 2016). Methods found were exploratory (Bonsaksen \& Lerdal, 2012; Browne et al., 2016; Firth et al., 2016), experimental (Oretel-Knochel et al., 2014; Strassnig et al., 2015; Toups et al., 2017), ethnographic (McArdle et al., 2012), prospective (Pfaff et al., 2014), pilot (Powers et al., 2015), and cross-sectional (Bonsaksen \& Lerdal, 2012; Subramaniapillai et al., 2016).

\subsection{Settings of Studies}

The settings took place in multiple countries around the world. Studies were based in Norway (Bonsaksen \& Lerdal, 2012), United States (Brown et al., 2015; Powers et al., 2015; Strassnig et al., 2015; Toups et al., 2017), Canada (Subramaniapillai et al., 2016), Australia (Pfaff et al., 2014), Germany (Oretel-Knochel et al., 2014), Ireland (McArdle et al., 2012), and the United Kingdom (Firth et al., 2016). Studies were conducted in different facilities. Some settings were inpatient based on a psychiatric unit (Bonsaksen \& Lerdal, 2012; Oretel-Knochel et al., 2014), outpatient (Browne et al., 2016; Firth et al., 2016; Subramaniapillai et al., 2016; Toups et al., 2017), physical training facilities (McArdle et al., 2012; Strassnig et al., 2015), home-based (Pfaff et al., 2014), and other settings otherwise not noted or vague in description (Powers et al., 2015). While the samples were not vague, there were a variety of sample sizes and diagnoses that were included.

\subsection{Sample Sizes of Studies}

Studies included sample sizes from $(\mathrm{N}=9)$ the smallest sample (Powers et al., 2015) to $(\mathrm{N}=200)$ the largest sample of subjects (Pfaff et al., 2014) giving a mean sample size for the review of 64.2. Some studies had an even representation of male and female subjects while others had skewed numbers of males (McArdle et al., 2012) and females (Powers et al., 2015). Other sample focus was at the client and clinicians (Browne et al., 2016). Mental illness diagnoses were a focus of the sample groups for the reviewed studies, which included depression or major depressive disorder (MDD), anxiety, schizophrenia, bipolar disorder, and posttraumatic stress disorder (PTSD). Six of the articles were related to depression and MDD (Bonsaksen \& Lerdal, 2012; Browne et al., 2016; McArdle et al., 2012; Oretel-Knochel et al., 2014; Pfaff et al., 2014; Toups et al., 2017) and one PTSD (Powers et al., 2015). Exercise as an intervention was found to be beneficial in six studies for treating mental illnesses (Firth et al., 2016; McArdle et al., 2012; Oretel-Knochel et al., 2014; Powers et al., 2015; Strassnig et al., 2015; Toups et al., 2017) along with other related subthemes.

\subsection{Other Themes of Studies}

Other major themes found in the reviews were preference such as exercise choice, exercise structure, and exercise length (Browne et al., 2016; McArdle et al., 2012; Subramaniapillai et al., 2016), which helped improve compliance in one study (McArdle et al., 2012). Another theme found is symptom severity (Bonsaksen \& Lerdal, 2012; Firth et al., 2016; McArdle et al., 2012; Pfaff et al., 2014; Powers et al., 2015) and exercise was effective in reducing mental illness symptom severity in three studies (Firth et al., 2016; McArdle., 2012; Powers et al., 2015). Cognitive ability was a predominant sub-theme that was found as well and effectively treated with exercise (Oretel-Knochel et al., 2014; Strassnig et al., 2015).

\subsection{Measurement Tools Used in Studies}

Measurements of major variables were completed with some strategies that included multiple measurement tools. The Diagnostic and Statistical Manual is a tool that was used to measure the severity of depression (Pfaff et al., 2014). Other measurements of anxiety and depression were measured with the hospital anxiety and depression scale ([HADS], Bonsaksen \& Lerdal, 2012). Depression measurements were conducted with the motivation energy inventory ([MEI], Bonsaksen \& Lerdal, 2012) and Center for Epidemiologic Studies depression scale ([CES-D], Smith-Marek et al., 2017). Trauma was measured with the life event checklist for DSM-5 (LEC-5). Measures of cognitive training and performance were captured by using the brief assessment of cognition in schizophrenia (BACS), which would also be used to help measure items such as symbol coding (Oertel-Knochel et al., 2014). There were many other forms of measure and measurement tools found in the ROL that are listed in Table 2. 
Table 2. Primary research for aerobic exercise and the effects on mental illness

\begin{tabular}{|c|c|c|c|c|c|c|c|c|}
\hline Citation & $\begin{array}{l}\text { Conceptual } \\
\text { Framework } \\
\end{array}$ & Design/Method & $\begin{array}{l}\text { Sample/ } \\
\text { Setting }\end{array}$ & $\begin{array}{c}\text { Major Variables } \\
\text { Studied and } \\
\text { Definitions } \\
\end{array}$ & $\begin{array}{l}\text { Measurement of } \\
\text { Major Variables }\end{array}$ & $\begin{array}{c}\text { Data } \\
\text { Analysis }\end{array}$ & $\begin{array}{c}\text { Study } \\
\text { Findings }\end{array}$ & $\begin{array}{c}\text { Study } \\
\text { strengths } \\
\text { and } \\
\text { weaknesses }\end{array}$ \\
\hline $\begin{array}{l}\text { Bonsaksen \& } \\
\text { Lerdal, } \\
(2012)\end{array}$ & $\begin{array}{l}\text { Self-reporting } \\
\text { physical } \\
\text { activity, } \\
\text { depression, } \\
\text { anxiety, and } \\
\text { quality of life }\end{array}$ & $\begin{array}{l}\text { - Quantitative } \\
\text { Cross-sectional, } \\
\text { correlational } \\
\text { study } \\
\text { - Exploratory }\end{array}$ & $\begin{array}{l}\text { Sample } \mathrm{N}=18 \\
\text { - Inpatient } \\
\text { clients with } \\
\text { severe } \\
\text { mental } \\
\text { illness } \\
\text { - Male ( } \mathrm{n}= \\
\text { 12) } \\
\text { - Female ( } \mathrm{n}= \\
6 \text { ) } \\
\text { - Mean age } \\
43.7 ; \mathrm{SD}= \\
13.3 \\
\text { Setting } \\
\text { - DP OUH } \\
\text { - Norway }\end{array}$ & $\begin{array}{l}\text { - Exercise } \\
\text { - Anxiety/Depression } \\
\text { - Quality of Life } \\
\text { - Functioning }\end{array}$ & $\begin{array}{l}\text { GAF } \\
\text { - Scale 0-100; } \\
\text { 21-30 = severe } \\
\text { problems } \\
\text { - Measures } \\
\text { Functioning } \\
\text { - Divided into } 2 \\
\text { sub-scales; } \\
\text { symptoms and } \\
\text { functional ability } \\
\text { IPAQ } \\
\text { - Measures the } \\
\text { number of days } \\
\text { where } \\
\text { performance of } \\
\text { strenuous, } \\
\text { moderate, or } \\
\text { walking activity } \\
\text { and how long } \\
\text { each day } \\
\text { HADS } \\
\text { - } 14 \text {-item } \\
\text { questionnaire } \\
\text { measuring } \\
\text { anxiety and } \\
\text { depression } \\
\text { - Likert scale; }(0) \\
\text { = occasionally to } \\
\text { (3) very often } \\
\text { - Score range } \\
0-21 ;>11= \\
\text { depression or } \\
\text { anxiety; > 19= } \\
\text { mixed } \\
\text { anxiety/depressio } \\
\text { BREF } \\
\text { - } 26 \text {-item quality } \\
\text { of life } \\
\text { questionnaire } \\
\text { pour domains: } \\
\text { physical, } \\
\text { psychological, } \\
\text { social relations, } \\
\text { and environment } \\
\text { 0-100 scale }\end{array}$ & 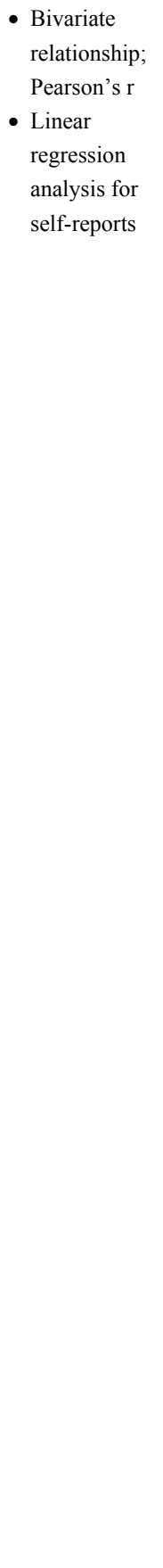 & $\begin{array}{l}\text { GAF } \\
\text { - Symptoms: } \\
\mathrm{M}=33.9 \\
\text { - Functioning } \\
\mathrm{M}=34.6 \\
\text { IPAQ } \\
\text { - }(\mathrm{n}=4 ; 22 \%) \\
\text { Strenuous } \\
\text { activity, } \\
\text { (n=9; } 50 \%) \\
\text { moderate } \\
\text { activity, } \\
(\mathrm{n}=15 ; \\
93.3 \%) \\
\text { walking } \\
\text { HADS } \\
\text { - Anxiety M = } \\
10.5 ; \mathrm{SD}= \\
4.9 \\
\text { - Depression } \\
\mathrm{M}=10.3 ; \\
\mathrm{SD}=4.6 \\
\text { BREF } \\
\text { - Total M = } \\
50.2 ; \mathrm{SD}= \\
18.5\end{array}$ & $\begin{array}{l}\text { Strengths: } \\
\text { - Used } \\
\text { multiple } \\
\text { validated } \\
\text { tools for } \\
\text { data } \\
\text { - } 18 \text {-month } \\
\text { study } \\
\text { - Risk was } \\
\text { low } \\
\text { Weakness: } \\
\text { - Quality of } \\
\text { evidence is } \\
\text { questionabl } \\
\text { e } \\
\text { - Small } \\
\text { sample } \\
\text { - One setting } \\
\text { No follow up }\end{array}$ \\
\hline $\begin{array}{l}\text { Browne et al. } \\
(2016)\end{array}$ & $\begin{array}{l}\text { An exploration } \\
\text { of client and } \\
\text { clinician } \\
\text { perspectives on } \\
\text { exercise and } \\
\text { possible } \\
\text { barriers with } \\
\text { focus on } \\
\text { walking groups }\end{array}$ & $\begin{array}{l}\text { - Mixed-method } \\
\text { Design } \\
\text { - Focus groups of } \\
\text { clients and } \\
\text { clinicians }\end{array}$ & $\begin{array}{l}\text { Sample } \mathrm{N}=26 \\
\text { - Clients } \\
(\mathrm{n}=12) \\
\text { - Diagnosed } \\
\text { with } \\
\text { schizophreni } \\
\text { a, bipolar, or } \\
\text { major } \\
\text { depression }\end{array}$ & $\begin{array}{l}\text { - Exercise } \\
\text { - Barriers } \\
\text { - Walking group } \\
\text { - Incentives to exercise }\end{array}$ & $\begin{array}{l}\text { Client } \\
\text { questionnaire } \\
\text { - Rate their } \\
\text { activity, comfort } \\
\text { with pedometer, } \\
\text { and walking } \\
\text { group } \\
\text { Clinician } \\
\text { questionnaire }\end{array}$ & $\begin{array}{l}\text { Questionnaire } \\
\text { - Two raters } \\
\text { to read } \\
\text { transcript, } \\
\text { code data, } \\
\text { decipher any } \\
\text { substantive } \\
\text { differences } \\
\text { - Multiple }\end{array}$ & $\begin{array}{l}\text { Exercise } \\
\text { - both } \\
\text { identified } \\
\text { similar } \\
\text { reasons to } \\
\text { exercise } \\
\text { - differing } \\
\text { views of the } \\
\text { client's }\end{array}$ & $\begin{array}{l}\text { Strength: } \\
\text { - Perspective } \\
\text { of both } \\
\text { clinician } \\
\text { and client } \\
\text { - Use of } \\
\text { feedback to } \\
\text { solidify } \\
\text { interest in }\end{array}$ \\
\hline
\end{tabular}




- Ages 25-50;
$\mathrm{M}=39.7 ;$
$\mathrm{SD}=7.7$
- Clinicians
$(\mathrm{n}=14)$
- Master's
level social
workers
- Ages $24-55 ;$
$\mathrm{M}=37.3 ;$
$\mathrm{SD}=10.1$
Setting
- Outpatient
clinic in two
cities
South Eastern
state

Firth et al. (2016)

\begin{tabular}{|c|c|c|c|}
\hline $\begin{array}{l}\text { Qualitative } \\
\text { method to } \\
\text { discuss } \\
\text { incentives, } \\
\text { barriers, and } \\
\text { opinions to } \\
\text { exercise and } \\
\text { optimal } \\
\text { interventions }\end{array}$ & $\begin{array}{l}\text { - } \\
\text { Exploratory-desc } \\
\text { riptive of the } \\
\text { iBeep feasibility } \\
\text { trial } \\
\text { - } 10 \text {-week } \\
\text { intervention } \\
\text { - Achieve } \geq 90 \\
\text { min of } \\
\text { moderate-to-vigo } \\
\text { rous activity } \\
\text { each week } \\
\text { - Follow up } \\
\text { interviews }\end{array}$ & $\begin{array}{l}\text { Sample: N=19 } \\
\text { interviewees } \\
\text { - (n=9) } \\
\text { interviews } \\
\text { immediately } \\
\text { post } \\
\text { program } \\
\text { - (n=10) } \\
\text { interviews at } \\
\text { 6-month } \\
\text { follow up } \\
\text { - (n=6) } \\
\text { subgroup } \\
\text { follow up of } \\
\text { both } \\
\text { immediate } \\
\text { and 6-month } \\
\text { - Recruited } \\
\text { from EIP } \\
\text { services in } \\
\text { Greater } \\
\text { Manchester, } \\
\text { UK } \\
\text { Setting: } \\
\text { - Community } \\
\text { leisure } \\
\text { schemes' } \\
\text { - Commonpla } \\
\text { ce } \\
\text { throughout } \\
\text { the UK }\end{array}$ & $\begin{array}{l}\text { - Individuals in early } \\
\text { stages of psychosis } \\
\text { - Which exercise was } \\
\text { of benefit or hindered } \\
\text { psychosis } \\
\text { - Exercise }\end{array}$ \\
\hline
\end{tabular}

- How active
clients are, is
there a need for
feasible exercise,
would refer to
walking group
Focus groups
examining
- exercise,
barriers,
incentives, and
attitudes about
walking groups
- All ratings used a
Likert scale for
feedback; (1) not
at all to (5) very

Semi-structured

interviews

- Discuss

incentives and

barriers to

exercise

- Short and long

term effect

- Opinions on

optimal

interventions

- Topic guides

- Interviews

conducted by

different RA than

participated in

exercise

five stages

of

qualitative

analysis

(FSQA)

- Re-read

- Generated

codes and

indexed

- Determine prevalent

themes

$\begin{array}{ll}\text { - Differing } & \text { walking } \\ \text { view on the } & \text { - Low rogram } \\ \text { reason to } & \text { - Feasible to } \\ \text { exercise } & \text { use in } \\ \text { (client=better } & \text { practice } \\ \text { mood, } & \text { Weakness: } \\ \text { clinician=tra } & \text { - Low } \\ \text { nsportation) } & \text { sample N } \\ \text { Barriers } & \text { - Only one } \\ \text { - Client same } & \text { facility } \\ \text { as to public } & \text { - 4-month } \\ \text { motivation } & \text { duration }= \\ \text { - Clinician } & \text { limited } \\ \text { believes } & \text { time } \\ \text { symptom } & \\ \text { related } & \end{array}$

Walking group

- Highly interested

(Both)

Incentives to

exercise

Social

interaction,

tangible

motivation

(pedometer),

- Length of

time of

exercise: $\mathrm{M}$

$=119 \mathrm{~min}$. of

moderate to

vigorous

exercise

Three

- Applied the

- Internal

homogeneit

$\mathrm{y}$ and

external

heterogeneit

$\mathrm{y}$, similar

themes

determine

links

- Themes

analyzed for

overarching

themes

- Exercise

alleviating

psychiatric

symptoms

- Improved

self-percepti

on

- Factors

determining

exercise

participation

Strengths:

- Longitudin

al study

with

interviews

right after

interventio

$\mathrm{n}$ and 6

months

later

- Use of

different

RA's to do

interviews

than did

exercise

- FSQA

- Subject perspective of

quantitativ e research

Weakness:

- Study from one area limiting culture

- $92 \%$ male 


\begin{tabular}{|c|c|c|c|c|}
\hline $\begin{array}{l}\text { McArdle et } \\
\text { al. (2012) }\end{array}$ & $\begin{array}{l}\text { Qualitative } \\
\text { methods to } \\
\text { document the } \\
\text { experiences of } \\
\text { participants of } \\
\text { the BTN } \\
\text { portion of the } \\
\text { pilot study }\end{array}$ & $\begin{array}{l}\text { - Ethnographic } \\
\text { research/ } \\
\text { Interviewing } \\
\text { participants post } \\
\text { BTN } \\
\text { intervention }\end{array}$ & $\begin{array}{l}\text { Sample: } \mathrm{N}=15 \\
\text { - }(\mathrm{n}=9) \text { focus } \\
\text { group } \\
\text { - }(\mathrm{n}=6) \\
\text { individual } \\
\text { phone } \\
\text { interview } \\
\text { - Men ages } \\
\text { 18-40 } \\
\text { Setting: } \\
\text { - Ireland }\end{array}$ & $\begin{array}{l}\text { - Men not seeking help } \\
\text { from formal health } \\
\text { care for psychological } \\
\text { distress } \\
\text { - Feeling of enjoyment } \\
\text { - Mastery } \\
\text { - Positive affect } \\
\text { - Teamwork } \\
\text { - Problem solving }\end{array}$ \\
\hline
\end{tabular}

Researcher
developed topic
guide
- Group discussion
- Asked number of
questions,
open-ended
Further
development
questions
- Individual phone
interview
- More topics that
researchers felt
warranted from
groups
Overall topic guide
- Covered 5 topics
related to BTN
triangulation to reduce bias

- Interpretive thematic analytic approach

- Member checking post data collection for

clarification with subject

- Audio recording of group discussions and individua interviews

- First and second author independent ly analyzed data
Core structural Strength

features:

- Cooperative ethos;

Participants felt comfortable in the environment.

Less competitive than anticipated.

- Central

ideas of importance Emphasizi ng cooperatio $\mathrm{n}$, cooperatio $\mathrm{n}$ instead of competitio $\mathrm{n}$, longer sessions modalities; $\quad$ (>55min), The health different promotion of times for CBT was group talk integrated Weaknesses into the sport - Small of football, sample less with only perceived completers stigma Impact of a program combined Possible exercise/CBT positive program on bias participants' experiences:

- Therapeutic experience; Having the "craic" or good enjoyment with the lads

- Anticipation to going to event was good

- Increased self-care, better time management, increase in pleasant

- A feeling of respite from daily life

- Able to use group as a means to talk Extended impact

- Disappointm ent that program ended at 10 weeks

- Participants 


$\begin{array}{lll}\begin{array}{l}\text { Oertel-Knoch } \\ \text { el et al. }\end{array} & \text { To } & \text { Q } \\ \text { understand } & \text { method } \\ \text { t2014) } & \text { the effects of } & \bullet \text { Experimental } \\ & \text { exercise } & \text { design } \\ & \text { across } & \bullet 4 \text { weeks } \\ & \text { disease } & \\ & \text { groups, } & \\ & \text { understand } \\ & \text { disease } \\ & \text { specific } \\ & \text { versus } \\ & \text { general or } \\ & \text { overlapping } \\ & \text { effects of } \\ & \text { physical } \\ & \text { training }\end{array}$

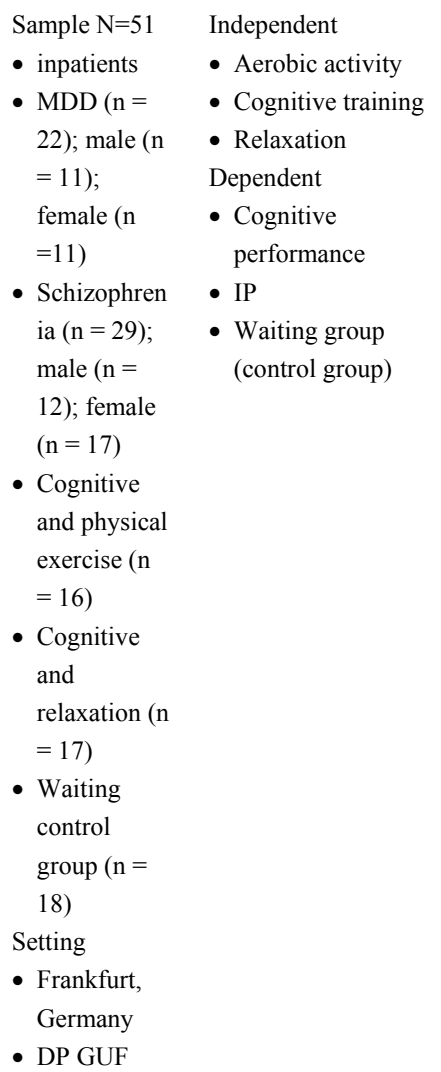

joined other

sports to

keep activity

and

socialization

Cognitive

performance

TMT A

- Psychomotor speed

BACS SC

- Assessment of cognition $\mathrm{r} / \mathrm{t}$ symbol coding

WMS-III SS

- Working memory and spatial span

- LNS

HVLT-R

- Fluency: animal naming and verbal learning BVMT-R

- Visual learning STAI

- Measuring state anxiety

- 20 item test

- Subjects instructed to rate anxiety

- to rate anxiety with scores ranging from 20-80; higher score $=$ higher anxiety SF-12: PSK

- Mental health subscale

- Score; $0=$ low subjective health, $100=$ high subjective health PANSS

- Semi structured interview

- Measurement of psychosis symptoms

Anxiety
- 4-point
Likert scale
LNS;
WMS-III SS;
TMT A;
BACS SC;
HVLT-R;
BVMT-R;
STAI
- ANCOVAs
SF-12: PSK
- Nonparamet
ric
Wilcoxon
test

Speed

Processing

- Increased in all groups

(Time: F (46)

$=37.55$; $\mathrm{p}<$

0.001 )

Working

Memory

- Decrease in

MDD but

increase in

all other

groups

(Intervention

group: F (46)

$=10.32 ; \mathrm{p}=$

0.02 )

Verbal

Learning

- Increased in

all groups

$(\mathrm{p}>0.05)$

Visual Learning

- $(\mathrm{F}(46)=$ 9.53; $\mathrm{p}=$ 0.004)

STAI

- Time (F (46) $=6.06 ; \mathrm{p}=$ 0.02 )

- SF-12

- (Time: F (46) $=18.62, \mathrm{p}<$ 0.001 )

- BDI II

- Depressive symptoms (F (19) $=24.10$; $\mathrm{p}<0.001$ )

- PANSS

Negative ( $F$

(26) = 8.34;

$\mathrm{p}=0.02$ )

- PANSS

Positive no

change ( $\mathrm{p}>$

0.05)

- Power analysis: high effect for visual learning $(\mathrm{d}=$ 0.91 ) for exercise; low effect for of Strengths

- Multiple

Valid

assessment

tools

- Control

group

- Multiple groups comparing different approaches for Tx

- Low risk of harm

- Feasible in practice

Limitations

- Short interventio $\mathrm{n}$ period

- Use/Chang es of medication s skew findings

- Use of PANSS (clinician use) and BDI II (subjective info) then comparing the two different diseases with different scales 


\begin{tabular}{|c|c|c|c|c|}
\hline $\begin{array}{l}\text { Paff et al. } \\
(2014)\end{array}$ & $\begin{array}{l}\text { Focus on } \\
\text { decreasing } \\
\text { depressive } \\
\text { symptoms of } \\
\text { older adults }\end{array}$ & $\begin{array}{l}\text { - Randomized } \\
\text { controlled trial } \\
\text { - Prospective, two } \\
\text { group parallel } \\
\text { - } 12 \text {-week } \\
\text { home-based } \\
\text { program to meet } \\
\text { guidelines for } \\
\text { people } 65 \text { and } \\
\text { older } \\
\text { - Monitored for } 52 \\
\text { weeks }\end{array}$ & $\begin{array}{l}\text { Sample: } \\
\text { - N=200 } \\
\text { adults } \geq 50 \\
\text { years of age } \\
\text { suffering } \\
\text { from a } \\
\text { clinically } \\
\text { depressive } \\
\text { illness } \\
\text { - Usual care } \\
\text { (n=92) } \\
\text { - Exercise } \\
\text { program } \\
\text { (n=108) } \\
\text { Setting: } \\
\text { - Community- } \\
\text { based } \\
\text { - Perth-metro } \\
\text { politan } \\
\text { region of } \\
\text { Western } \\
\text { Australia }\end{array}$ & $\begin{array}{l}\text { - IV=Usual medical } \\
\text { care plus physical } \\
\text { activity } \\
\text { - DV1=Severity of } \\
\text { depression } \\
\text { - DV2=Change in } \\
\text { physical activity } \\
\text { - Control=usual care }\end{array}$ \\
\hline
\end{tabular}

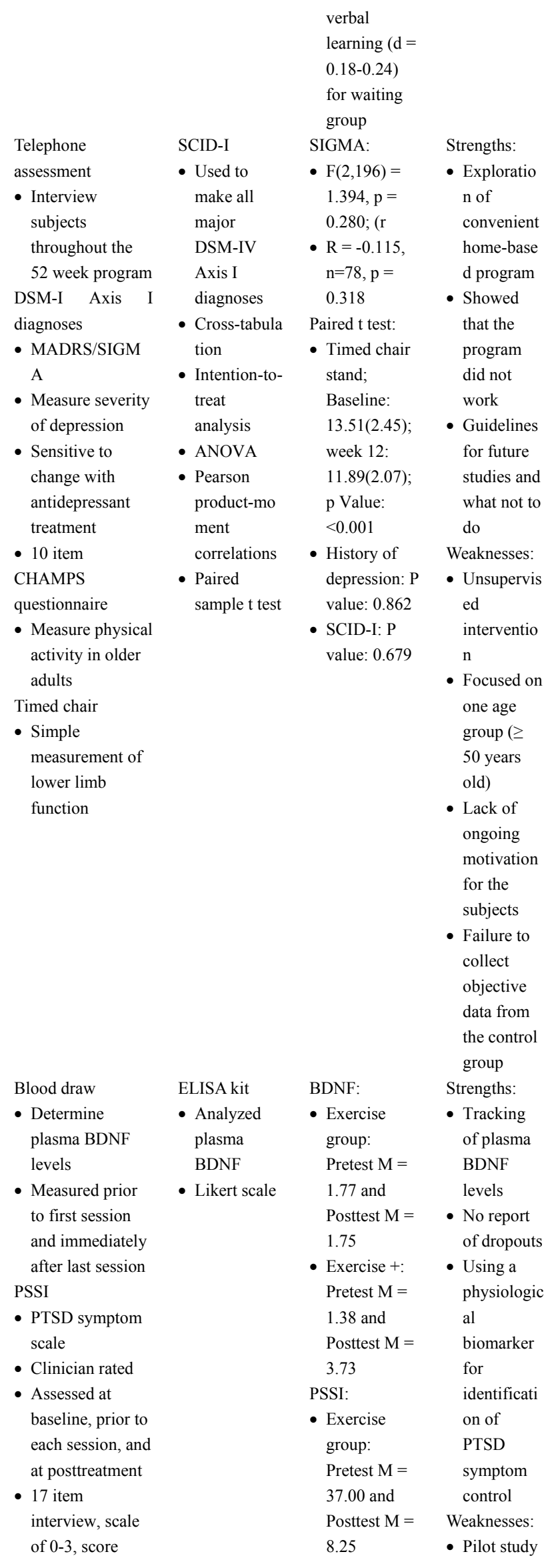




\begin{tabular}{|c|c|}
\hline $\begin{array}{l}\text { Strassnig et } \\
\text { al. (2015) }\end{array}$ & $\begin{array}{l}\text { - Study to } \\
\text { focus on } \\
\text { changes in } \\
\text { ADL } \\
\text { abilities } \\
\text { - Self-care } \\
\text { deficit } \\
\text { nursing } \\
\text { theory } \\
\text { (Orem, } \\
\text { 2001) }\end{array}$ \\
\hline
\end{tabular}

\begin{tabular}{|c|c|c|}
\hline $\begin{array}{l}\text { - Quantitative, } \\
\text { Quasi-Experime } \\
\text { ntal Research } \\
\text { 8-week study of } \\
\text { patients with } \\
\text { schizophrenia } \\
\text { and bipolar to } \\
\text { study acceptance } \\
\text { and feasibility of } \\
\text { high velocity } \\
\text { circuit training }\end{array}$ & $\begin{array}{l}\text { Sample: } \\
\text { N=12 } \\
\text { - Male ( } \mathrm{n}=9 \text { ) } \\
\text { - Female ( } \mathrm{n}= \\
\text { 3) } \\
\text { - Overweight/ } \\
\text { Obese } \\
\text { - Bipolar } \\
\text { (n=3) } \\
\text { - Schizophren } \\
\text { ia (n=9) } \\
\text { - Ages 18-75 } \\
\text { Setting: } \\
\text { - State of the } \\
\text { art training } \\
\text { facility at } \\
\text { UMMSM } \\
\text { - Community } \\
\text { dwelling }\end{array}$ & $\begin{array}{l}\text { - PNSS/Bipolar } \\
\text { Strength } \\
\text { - 1RM } \\
\text { Power } \\
\text { - Rated by the highest } \\
\text { value of repetitions } \\
\text { and loading } \\
\text { conditions } \\
\text { Anthropometric } \\
\text { measures } \\
\text { - Measurements of the } \\
\text { body composition } \\
\text { Physical function and } \\
\text { ADL assessments } \\
\text { - Ability to complete } \\
\text { ADL tasks and have } \\
\text { physical ability of } \\
\text { gait, balance, and } \\
\text { lower extremity } \\
\text { function }\end{array}$ \\
\hline
\end{tabular}

range $0-51$;

higher score $=$

more severe

PTSD

Exercise + :
Pretest $\mathrm{M}=$
42.00 and
Posttest $\mathrm{M}=$
5.20

PNSS

- Positive,

negative, and

general

schizophrenic

symptoms

- Used to gather

baseline of

positive and

negative

schizophrenic

symptoms

BACS

- assessment of

cognition

- Verbal memory, digit sequencing, verbal fluency,

symbol coding,

composite scores

- Used shortened

version to reduce

time

CDS

- Measurement of

depression

SPPB

- Function and performance of ADL assessment

- Examine lower extremity

performance,

e.g., rise from

chair

performance

PFP-10

- Measurement of upper extremity strength and

flexibility, lower extremity strength, balance, coordination

Strength

- 1RM

- Power tested at $30 \%$ to $90 \%$ of 1RM load,

- Perform as quickly as possible
- Small

sample

- Only 12

sessions

- Low

generalizati

on

Paired t-test

Strength

- 1RM, Chest

press: $(\mathrm{p}=$

$0.008 ; \mathrm{F}=$

$10.481 ; \mathrm{n} 2=$

0.488 )

Power

ANOVA

- Neuromuscu

- Leg

lar

- $\mathrm{BC}$

- DFV

Significance

- P value set at 0.05

- Effect size $\mathrm{n} 2$

computed

for all

variables

extension: ( $p$

$=0.003 ; \mathrm{F}=$

$14.50 ; \mathrm{n} 2=$

0.569 )

BAC T scores

- Composite:

Pretest: 32.3

to Posttest:

35.4; $(\mathrm{t}=$

$-3.3 ; \mathrm{df}=11$;

$\mathrm{p}=0.008)$

- Effect size: d $=0.48$

PNSS T scores

- Total:

Pretest: 58.6

to Posttest:

54.3; $(\mathrm{t}=$

$2.5 ; \mathrm{df}=11$;

$\mathrm{p}=0.03$ )

CDS T scores

- Pretest: 4.8

to Posttest:

$3.2 ;(\mathrm{t}=2.6$;

$\mathrm{df}=11 ; \mathrm{p}=$

0.025 )
- Evidence was valid but no control group, small sample size, and lack of intention to treat analysis.

- scores on

PNSS and depression or CG may be biases

- Need for scale that is appropriate for a younger age group for

- No dietary monitoring or interventio ns

- Need for longer training period

- More days/wk 


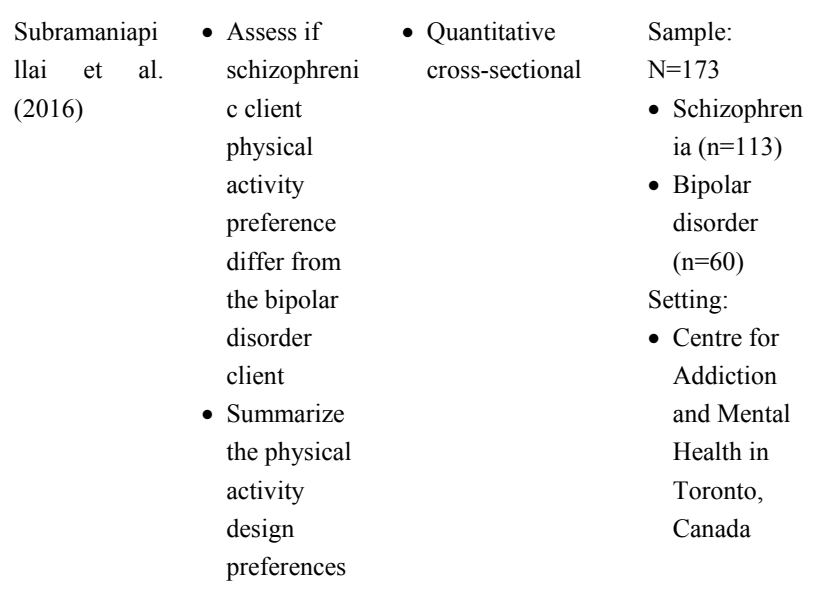

- Major diagnoses of schizophrenia and bipolar

Two-part questionnaire

- Personal preferences

- Preferred program structure

(2)

\begin{tabular}{|c|c|c|}
\hline $\begin{array}{l}\text { Toups et al. } \\
\text { (2017) }\end{array}$ & $\begin{array}{l}\text { Evaluated } \\
\text { the effect of } \\
\text { exercise on } \\
\text { positive } \\
\text { valence } \\
\text { symptoms of } \\
\text { MDD as part } \\
\text { of the } \\
\text { TREAD } \\
\text { program for } \\
\text { clients } \\
\text { taking a } \\
\text { stable dose }\end{array}$ & $\begin{array}{l}\text { - Experimental } \\
\text { - } 12 \text {-week } \\
\text { - Exercise } \\
\text { program }\end{array}$ \\
\hline
\end{tabular}

$\begin{aligned} & \text { Stages of change } \\ & \text { algorithm }\end{aligned}$
- Self-characterizat
analysis
ion of physical
activity
MINI
- Confirian split
diagnoses
- Descriptive
statistics:
percentages
Physical activity
questionnaire short
form
- Two part:
Preferences;
Preferred
structure of
exercise
- How much
moderate to
vigorous
intensity
completed in the
last week

Diagnoses: Strength:

- $66.4 \%$ - Gaining

schizophreni client

a; $30.7 \% \quad$ perspective

schizoaffecti - Approved

ve; $2.1 \% \quad$ by research

psychosis and ethics

NOS; board at

Bipolar: the

$36.7 \% \quad$ University

bipolar I; of Toronto,

$60.0 \%$ - Specific

bipolar II; questions

$3.3 \%$ bipolar to add

NOS quantitativ

Chi square: e data

- No

significant

differences

Stages of

changes:

- Schizophreni

a: $48.7 \%$

preparation

stage

- Bipolar:

$33.3 \%$

preparation

stage

Weaknesses:

- Questions were

narrowed

with no

room for

elaboration

of

preferences

- Need for

more

research to

strengthen

findings

- Only two

mental

illnesses

screened

$\begin{array}{ll}\text { Sample: } & \text { • Valence symptoms } \\ \mathrm{N}=119 & \text { - MDD severity } \\ \text { - High dose } & \text { - Baseline level of } \\ (\mathrm{n}=119) ; & \text { fitness } \\ \text { female } & \text { - Inventory of } \\ (84.5 \%) & \text { depression } \\ \text { - Low dose } & \text { symptomatology } \\ (\mathrm{n}=61) ; & \\ \text { female } & \\ (78,7 \%) & \\ \text { Setting: } & \\ \text { - Cooper } & \\ \text { Institute in } & \\ \text { Dallas, TX } & \\ \text { for initial } & \\ \text { two weeks } & \\ \text { - Remaining } & \\ \text { program at } & \\ \text { home } & \end{array}$

SHAPS

- Measuring

positive valence

symptoms

- Used to evaluate the effect of

exercise on

MDD

- Score $>2=$

clinically

abnormal

MEI

- Measuring positive valence

- Used to evaluate effect of exercise on MDD

- Measure:

physical energy, mental energy,

social motivation

- 27 item, scored

$0-6$, range 0-162

and higher score

$=$ higher energy

and motivation
- ROC

analysis

- Likert Scale

- SAS PROC

MIXED

- Spearman's correlation

- Path

analysis

- Cross-lagge d panel model using MPLUS

Version 7.3

- Significance 0.05
Baseline level Strengths:

of fitness: - 12-week

- Episode/Mon study

th: All mean - Showing

$=81.5(96.8) \quad$ significant

- MEI total improveme

score: All nt in

mean $=$ depression

34.7(14.4) - Supports

- SHAPS total the use of

score: All TREAD

mean $=\quad$ Weaknesses:

3.1(3.0) - Exclusion

Post

of many

intervention:

- SHAPS at 6

weeks: All

mean total $=$

$-0.81=2.29$

total

- MEI at 6

weeks: All

mean total $=$

$9.96=44.66$

total

clients that

suffer from

MDD

- Already

stable on

SSRI

- No power analysis

- Low generalizati on

- SHAPS at 12 weeks: All 


$\begin{array}{ll}\text { - Measurement of } & \text { mean total }= \\ \text { overall MDD } & -1.19= \\ \text { severity } & 1.91 \text { total } \\ & \text { - MEI at } 12 \\ & \text { weeks: All } \\ \text { mean total }= \\ 16.08= \\ 50.78 \text { total } \\ \text { P-value for All } \\ \text { at week } 12= \\ 0.054\end{array}$

Note: Activities of Daily Living (ADL); Back of the Net (BTN); Body Composition (BC); Brief Assessment of Cognition in Schizophrenia (BACS); Brief Assessment of Cognition in Schizophrenia: Symbol Coding (BACS SC); Center for Epidemiologic Studies Depression Scale (CES-D); Community Healthy Activities Model Program for Seniors (CHAMPS); Clinical Global (CG); Cognitive Behavior Techniques (CBT); Calgary Depression Scale (CDS); Daily Function Variables (DFV); Department of Psychiatry (DP); Five Stages of Qualitative Analysis (FSQA); Germany (GER); Global Assessment of Functioning Scale (GAF); Goethe-University, Frankfurt (GUF); High Velocity Circuit Resistance Training (HVCR); Hospital Anxiety and Depression Scale (HADS); Inventory of Depression Symptomatology-Clinician Rated (IDS-C); Inventory of Depression Symptomatology-Self-Rated (IDS-SR); Investigating the Benefits of Exercise in Early Psychosis (iBeep); Individual Psychopathology (IP); International Physical Activity Questionnaire (IPAQ); Life Event Checklist for DSM-5 (LEC-5); Letter Number Span (LNS); Montgomery-Asberg Depression Rating Scale (MARDS); Medical Research Council (MRC); Mechanical Power (MP); Major Depressive Disorder (MDD); Mini International Neuropsychiatric Interview (MINI); Motivation and Energy Inventory (MEI); Non-completers (NC); Oslo University Hospital (OUH); One-repetition maximum (1RM); Patient Health Questionnaire (PHQ-9); Positive and Negative Symptom Score (PNSS); Posttraumatic Stress Disorder (PTSD); PTSD Symptom Scale-Interview (PSSI); Post Traumatic Stress Disorder Checklist (PCL-5); Power/1RM exercise include: Leg extension, chest press, overhead press, calf raise, lat pulldown, leg curl; Physical Functional Peromance-10 (PFP-10); Quality of Marriage Index (QMI); Quick Inventory of Depression Symptomatology (QIDS) Quick Inventory of Depression Symptomatology-Clinician (QIDS-C); Quick Inventory of Depression Symptomatology-Self-Rated (QIDS-SR); Receiver Operating Characteristic (ROC); Research Assistant (RA); Structured Clinical Interview for DSM-IV Axis I Disorders (SCID-I); Short Physical Performance Battery (SPPB); Snaith Hamilton Pleasure Scale (SHAPS); Symptoms and Cognition (SC); Total (T); Treatment with Exercise Augmentation for Depression (TREAD); Trial Making Test (TMT); Treatment (Tx); United Kingdom (UK); University of Miami Miller School of Medicine (UMMSM); World Health Organization Quality of Life (BREF)

\subsection{Diagnosis Specific Information}

Mental illness is a debilitating diagnosis that effects much more than the person's psyche but also the person's social abilities, loss of autonomy, and job-related problems (Oretel-Knochel et al., 2014). Exercise was found to be a very effective activity that positively influenced participants' mental health among those with the diagnoses of depression (Bonsaksen \& Lerdal, 2012; Browne et al., 2016; McArdle et al., 2012; Oretel-Knochel., 2014; Toups et al., 2017), anxiety (Bonsaksen \& Lerdal, 2012; Oretel-Knochel., 2014), schizophrenia disorders (Browne et al., 2016; Firth et al., 2016; Oretel-Knochel., 2014; Strassnig et al., 2015; Subramaniapillai et al., 2016), Bipolar (Browne et al., 2015; Strassnig et al., 2015; Subramaniapillai et al., 2016), and PTSD (Powers et al., 2015). There were significant improvements in subjects who suffer from schizophrenia disorders (Firth et al., 2016; Oretel-Knochel., 2014; Strassnig et al., 2015; Subramaniapillai et al., 2016). Positive (hallucinations and paranoia) and negative symptoms (amotivation and anhedonia) in schizophrenic subjects were shown to have symptom relief related to the intervention of exercise (Firth et al., 2016). Improvements in speed processing, working memory, verbal learning were found as a result of physical exercise and or relaxation therapy (Oertel-Knochel et al., 2014). High-velocity circuit resistance training (HVCR) can be beneficial for those who suffer from schizophrenia as well. HVCR improved the neuromuscular performance of subjects in an eight-week program. This program also had positive outcomes in cognitive scores including verbal memory, symbol coding, and verbal fluency addressed by the brief assessment of cognition (Strassnig et al., 2015). Depression was found to be relieved by the introduction of exercise as an intervention (Browne et al., 2016; Oretel-Knochel., 2014; 
Toups et al., 2017) and as an opportunity for socialization (McArdle et al., 2012).

Two studies dedicated to depression found opposite results with the intervention of exercise. Both studies were not located in an inpatient unit. One study was focused on an age group that was 50 years of age and greater and solely based at the subject's home (Pfaff et al., 2014). While the other study that had more positive results were using an age group ranging from 18-70 years of age, was well supervised in a training facility for the initial two weeks, and then continued the intervention at home (Toups et al., 2017). Subjects did poorer with the intervention solely in their home and supervision from a booster telephone call. The lack of observation perhaps led to the lack of intensity of the exercise interventions, which may have had an impact on the results. However, exercise was shown to have improvements in motivation as well as depressive severity that led to better social motivation for subjects (Toups et al., 2017). The exercise was also found to be beneficial in the enhancement of functioning and cognitive abilities (Oertel-Knochel et al., 2014; Strassnig et al., 2015).

The highest results of cognitive improvements and positive mood alterations were found in studies that had the usage of a facility with a directed program (Oertel-Knochel et al., 2014; Strassnig et al., 2015). This would include the use of on staff researchers, coaches, and physiologists to help encourage subjects to exert proper energy and complete the entire workout for the day (Oertel-Knochel et al., 2014; Strassnig et al., 2015). Programs were organized at clean state-of-the-art athletic facilities, which the subjects stated helped them continue the program (McArdle et al., 2012; Strassnig et al., 2015). The structured physical activities at a training facility (McArdle et al., 2012; Strassnig et al., 2015; Toups et al., 2017) and in an inpatient setting (Oretel-Knochel et al., 2014) were found to be more effective than the unobserved home-based programs (Pfaff et al., 2014).

Outcomes of the research were positive for the promotion of exercise as an intervention for mental illness (Firth et al., 2016; McArdle et al., 2012; Oretel-Knochel et al., 2014; Powers et al., 2015; Strassnig et al., 2015; Toups et al., 2017). The act of physical fitness showed a multi-layered positive effect for people who suffer from mental illness. There was an increased sense of well-being as well as an increased motivation for social interaction and a sense of excitement for the activity and increase in self-perception (Firth et al., 2016; McArdle et al., 2012; Oretel-Knochel et al., 2014; Powers et al., 2015; Strassnig et al., 2015; Toups et al., 2017). Exercise, in particular, aerobic exercise, can influence the BDNF plasma levels in the body and have a profound positive impact on PTSD symptom management (Powers et al., 2015). Other factors that influence a better quality of life were an increase in cognitive skills including working memory, verbal learning, and visual learning in subjects with schizophrenia (Oertel-Knochel et al., 2014). Exercise lowered symptoms of depression and anxiety while increasing daily functioning abilities even in inpatient adults with severe mental illness (Bonsaksen \& Lerdal, 2012). Other important outcomes are subject perspectives of the intervention and their perceived barriers to exercise (Browne et al., 2016). Some subjects found that exercise was very useful in relieving them of their daily stressors and looked forward to the activity (McArdle et al., 2012). While others in the early stages of psychosis found exercise therapeutic to their symptoms (Firth et al., 2016).

\section{Discussion}

Aerobic exercise is a beneficial intervention for people who suffer from mental illness and veterans are prone to mental health disorders with exposure to combat situations and high-stress environments (Pols \& Oak, 2007). Aerobic exercise was shown to have improvements in cognition and symptom management. Exercise as an intervention for veterans' post-military with acute mental health symptoms may show decreased severity of symptoms and may also reduce the frequency of acute exacerbations of chronic symptoms.

Exercise improves plasma levels of BDNF that aids in symptom management of PTSD, which is a rising mental health symptom of veterans who are returning from combat areas (Pols \& Oak, 2007). Mental health complications and symptoms are better controlled with the introduction of aerobic excise. The increased physical activity helps increase neuroplasticity helping the veteran in an acute mental health setting gain increased physiological pathways for increased neurological growth versus only using medication and therapy. Exercise can be used as a coping skill to help treat mental illness. An additional factor found to help motivate veterans to exercise was whether they were being cared for in an inpatient or outpatient setting.

The most beneficial setting in promoting active engagement in aerobic exercise was inpatient units with coaches. Coaching veterans in the inpatient mental health setting may be effective in increasing participation and gaining their feedback for program improvements. Veterans receiving mental health care may benefit from alternative therapies that help increase their level of healthy activity. Guidance to help combat the tendency for a sedentary lifestyle and antipsychotic medications increases susceptibility for weight gain as they can lead to other physical complications such as cardiovascular disease. 


\section{Limitations}

There were various limiting factors found in the reviewed studies. One of which was small sample sizes with a mean sample size of 63.3. Other limiting factors found were low generalization with interventions taking place at one facility. Some studies were skewed with a dominant presence of one sex in the study. Positive bias was found in a feedback study post intervention with a qualitative study and inclusion of only the completers of the intervention. Other interventions included the non-supervised approach to implementation leading to skewed data and non-compliance.

\section{Clinical Implications}

Staff can implement exercise as an intervention for patients in an inpatient mental health setting. Appropriate assessments, facility, and clearance from providers will be necessary to ensure safety for staff and patients. Staff can designate aerobic exercise as part of the veteran's treatment plan as they progress through the acute mental illness state to recovery. This would be most effective if led by a program supervisor to facilitate the exercise activities for the inpatients. The benefit for the veteran who participates could be lifelong, as exercise is a relatively inexpensive, learned intervention that they can continue as an outpatient.

\section{Recommendations for Further Research}

Further research would benefit with the exploration of aerobic activity and its effect on veterans in the inpatient setting through to the outpatient setting. Staff researchers and veterans would benefit from the retrieval of data from veteran patients that are admitted into the inpatient mental health setting and are introduced to aerobic activity as an aspect of their treatment plan. It will be beneficial to follow their progression through to recovery and compare with a control group of usual mental health recovery care. Researchers could continue the study into the outpatient setting and monitor the rates of return to the acute mental health setting between the intervention and control group to identify if the intervention group has an improved level of mental health recovery as compared to usual care.

\section{Conclusion}

Aerobic exercise is highly recommended for patients in the inpatient setting. This would be an intervention that could aid the veteran in mental health symptom management as well as physical symptoms that may accompany the disorder. Increases in activity would have a positive effect on the veteran's relationships and socialization opportunities. The veteran will be more apt to learn positive coping skills with exercise that is inexpensive and may reduce the chance of using substances to self-medicate (e.g., alcohol) and deter them from treatment.

\section{Acknowledgement}

The authors wish to acknowledge Dr. Kelly D. Ackerson, Bronson School of Nursing, Western Michigan University, Michigan, USA, for offering valuable comments and direction.

\section{References}

Bonsaksen, T., \& Lerdal, A. (2012). Relationships between physical activity, symptoms and quality of life among inpatients with severe mental illness. British Journal of Occupational Therapy, 75(2), 69-75. https://doi.org/10.4276/030802212x13286281651036

Browne, J., Mihas, P., \& Penn, D. L. (2016). Focus on exercise: Client and clinician perspectives on exercise in individuals with serious mental illness. Community Mental Health Journal, 52(4), 387-394. https://doi.org/10.1007/s10597-015-9896-y

Carter, T., Callaghan, P., Khalil, E., \& Morres, I. (2012). The effectiveness of a preferred intensity exercise programme on the mental health outcomes of young people with depression: A sequential mixed methods evaluation. BioMedical Central Public Health, 12(187), 1-9. https://doi.org/10.1186/1471-2458-12-187

Firth, J., Carney, R., Jerome, L., Elliott, R., French, P., \& Yung, A. R. (2016). The effects and determinants of exercise participation in first-episode psychosis: A qualitative study. BioMedical Central Psychiatry, 16(36), 1-9. https://doi.org/10.1186/s12888-016-0751-7

McArdle, S., McGale, N., \& Gaffney, P. (2012). A qualitative exploration of men's experiences of an integrated exercise/CBT mental health promotion programme. International Journal of Men's Health, 11(3), 240-257. https://doi.org/10.3149/jmh.1103.240.

Mojtabai, R. (2011). National trends in mental health disability, 1997-2009. American Journal of Public Health, 101(11), 2156-2163. https://doi.org/10.2105/AJPH.2011.300258

Oertel-Knochel, V., Mehler, P., Thiel, C., Steinbrecher, K., Malchow, B., Tesky, V ... Hansel, F. (2014). Effects of 
aerobic exercise on cognitive performance and individual psychopathology in depressive and schizophrenia patients. European Archives of Psychiatry and Clinical Neuroscience, 264, 589-604. https://doi.org/10.1007/s00406-0140485-9

Pfaff, J. J., Alfonso, H., Newton, R. U., Sim, M., Flicker, L., \& Almeida, O. P. (2014). ACTIVEDEP: A randomised, controlled trial of a home-based exercise intervention to alleviate depression in middle-aged and older adults. British Journal of Sports Medicine, 48(3), 226-232. https://doi.org/10.1136/bjsports-2013-092510

Pols, H., \& Oak, S. (2007). War \& military mental health the US psychiatric response in the 20th century. American Journal of Public Health, 97(12), 2132-2142. https://doi.org/10.2105/AJPH.2006.090910

Powers, M. B., Medina, J. L., Burns, S., Kauffman, B. Y., Monfils, M., Asmundson, G. J. G ... Smits, J. A. J. (2015). Exercise augmentation of exposure therapy for PTSD: rationale and pilot efficacy data. Cognitive Behavior Therapy, 44(4), 314-327. https://doi.org/10.1080/16506073.2015.1012740

Strassnig, M. T., Signorile, J. F., Potiaumpai, M., Romero, M. A., Gonzalez, C., Czaja, S., \& Harvey, P. D. (2015). High velocity circuit resistance training improves cognition, psychiatric symptoms and neuromuscular performance in overweight outpatients with severe mental illness. Psychiatry Research, 229, 295-301. https://doi.org/10.1016/j.psychres.2015.07.007

Subramaniapillai, M., Arbour-Nicitopoulos, K., Duncan, M., Mclntyre, R. S., Mansur, R. B., Remington, G., \& Faulkner, G. (2016). Physical activity preferences of individuals diagnosed with schizophrenia or bipolar disorder. BioMedical Central Research Notes, 9(340), 1-9. https://doi.org/10.1186/s13104-016-2151-y

Toups, M., Carmody, T., Greer, T., Rethorst, C., Grannemann, B., \& Trivedi, M. H. (2017). Exercise is an effective treatment for positive valence symptoms in major depression. Journal of Affective Disorders, 209, 188-194. https://doi.org/10.1016/j.jad.2016.08.058

Vazan, P., Golub, A., \& Bennett, A. S. (2013). Substance use and other mental health disorders among veterans returning to the inner city: Prevalence, correlates, and rates of unmet treatment need. National Institute of Health, 48(10), 880-893. https://doi.org/10.3109/10826084.2013.796989

World Health Organization. ([WHO], 2003). Investing in mental health. Department of Mental Health and Substance Dependence, Non-communicable Diseases and Mental Health. Retrieved from: http://www.who.int/mental_health/en/investing_in_mnh_final.pdf

\section{Copyrights}

Copyright for this article is retained by the author(s), with first publication rights granted to the journal.

This is an open-access article distributed under the terms and conditions of the Creative Commons Attribution license (http://creativecommons.org/licenses/by/4.0/). 